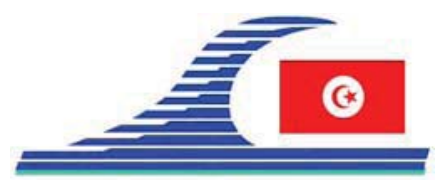

\author{
Conférence Méditerranéenne Côtière et Maritime \\ EDITION 1, HAMMAMET, TUNISIE (2009) \\ Coastal and Maritime Mediterranean Conference \\ Disponible en ligne - http://www.paralia.fr - Available online
}

\title{
Quid de l'impact de la déficience des données de base sur le choix des caractéristiques d'un émissaire en mer dans le golfe de Tunis?
}

\author{
Mathilde SION $^{1}$, Moncef BOUSSABAH ${ }^{1}$
}

1. GIC Tunisie Ingénieurs Conseils, 10 rue des Métiers, 2035 Tunis Carthage, Tunisie. sionmathilde@hotmail.com;gic@planet.tn

\begin{abstract}
:
When planning a sea outfall pipe design, the basic data gathering turns out to be a very fussy task because of the complexity of the marine environment. In case where basic data are not enough, With a theoretical approach, based on Stokes' model of heave, it is possible to obtain some information about the waves' dimensions, the breaking zone next to the seashore, and the drift phenomenon which is responsible for the pollutants' dissemination. But, almost of outfall pipe designers resort to numerical modelling tools to visualize sea currents and outflow evolution without required basic data. Waiting for reliable data gathering, we suggest to create a "Mediterranean Existing Outfall Pipe Observatory" that would provide more information about their functioning and give more help about future sea out fall pipe design.
\end{abstract}

\section{Mots-clés :}

Protection du littoral - Emissaire en mer -Dimensionnement - Houle - Dilution Modèles

\section{Préambule}

Plus le milieu naturel d'une zone de projet est complexe, plus la collecte des données de base relatives à l'effluent, la climatologie et le milieu marin se doit d'être complète et devient de ce fait difficile à effectuer. Ainsi l'essor du tourisme sur les côtes méditerranéennes et l'augmentation des volumes d'eau épurés à rejeter a rendu le recours aux émissaires de plus en plus systématique et que leur conception devra être une technique «bien rodée ». Par exemple, l'économie tunisienne dépend dans sa majeure partie de son littoral que l'Agence de Protection et d'Aménagement du Littoral est notamment chargée de sauvegarder. Cette communication entre dans le cadre du dimensionnement de l'émissaire en mer dans le golfe de Tunis et de l'étude menée par « GIC TUNISIE » pour l'Office National de Assainissement visant à supprimer le rejet actuel (près de 80 millions de $\mathrm{m}^{3}$ par an) de la station d'épuration de Choutrana sur la plage de Raoued. Cet émissaire est une composante du projet de transfert des eaux épurées du Grand Tunis vers des zones déficientes en eau du Kairouannais et servira

DOI: $10.5150 / \mathrm{cmcm} .2009 .056-5$ 
comme organe de secours du système hydraulique pour évacuer les eaux traitées en mer pour différentes raisons : excédent d'eau, régulation, accident épuratoire, etc.

Pour concevoir un émissaire en mer, il est nécessaire de déterminer les critères de choix des données de base utiles au projet, les données elles mêmes (voir tableau 1).

Tableau 1. Détermination des caractéristiques d'un émissaire en mer.

\begin{tabular}{|c|c|c|c|}
\hline & & \\
\hline & & Critère déterminant & Données requises \\
\hline \multirow{4}{*}{$\begin{array}{l}\text { Choix des } \\
\text { dimensions }\end{array}$} & $\begin{array}{l}\text { Profondeur à son } \\
\text { extrémité }\end{array}$ & $\begin{array}{l}\text { Minimiser les efforts exercés par les } \\
\text { courants }\end{array}$ & $\begin{array}{l}\text { Courantologie et } \\
\text { climatologie }\end{array}$ \\
\hline & $\begin{array}{l}\text { Profondeur du } \\
\text { tronçon à enterrer }\end{array}$ & Tronçon soumis au déferlement de la houle & $\begin{array}{l}\text { Courantologie et } \\
\text { climatologie }\end{array}$ \\
\hline & $\begin{array}{l}\text { Diamètre de la } \\
\text { conduite principale }\end{array}$ & $\begin{array}{l}\text { Critère hydraulique : minimiser le marnage } \\
\text { d'un bassin d'amortissement, les pertes de } \\
\text { charge, ... }\end{array}$ & $\begin{array}{l}\text { Caractéristiques des } \\
\text { équipements terrestres de } \\
\text { transport entre la STEP et } \\
\text { l'émissaire }\end{array}$ \\
\hline & Diffuseur & Dilution efficace de l'effluent & Etude de la dilution \\
\hline \multirow{3}{*}{$\begin{array}{l}\text { Dilution de } \\
\text { l'effluent }\end{array}$} & Phase de remontée & \multirow{3}{*}{$\begin{array}{l}\text { L'étude de la remontée du polluant et de sa } \\
\text { dilution progressive au cours de sa dérive } \\
\text { doit permettre de déterminer la distance } \\
\text { minimale entre le rejet et le littoral afin de } \\
\text { le préserver }\end{array}$} & Profil de densité du milieu \\
\hline & Phase de transport & & $\begin{array}{l}\text { Courantologie et } \\
\text { climatologie }\end{array}$ \\
\hline & Données communes & & $\begin{array}{l}\text { Dimensions de l'émissaire } \\
\text { Faune, flore, sédiments }\end{array}$ \\
\hline & & 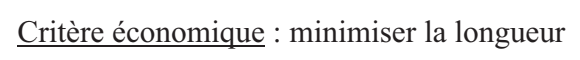 & \\
\hline Choix de & & totale de l'émissaire & Bathymétrie \\
\hline l'emplacement & & $\begin{array}{l}\text { Critère environnemental : atteindre une } \\
\text { longueur favorable à la dilution }\end{array}$ & Etude de la dilution \\
\hline
\end{tabular}

\section{Le problème de l'acquisition des données courantologiques}

La grande majorité des données climatologiques et terrestres sont disponibles en Tunisie où l'on dispose de séries de plus de 100 ans en ce qui concerne le milieu marin, les courants qui sont le résultat d'une juxtaposition très complexe de nombreux mouvements marins (houle, marées, courants généraux, déviation de Coriolis, ...) dont l'intensité, la profondeur et la direction sont difficilement prédictibles et par conséquent on dispose de très peu de données hormis celles concernant les courants de houle et de surface qui peuvent suffire en Méditerranée, pour ce type de projets.

Compte tenu de l'absence de mesures de profils courantologiques sur des périodes relativement longues, au moins une année, et afin de disposer d'informations relatives aux phénomènes de la houle dans la zone du projet, en l'occurrence le golfe de Tunis, deux options majeures s'offrent aux concepteurs : le recours aux équations empiriques de la houle et l'utilisation systématique de modèles mathématiques. 


\section{Le modèle de la houle de Stokes}

L'étude du phénomène de la houle et de sa dérive, permet d'une part, d'estimer les caractéristiques dimensionnelles de l'émissaire (longueurs et profondeurs) et d'autre part d'estimer le devenir des particules en suspension rejetées à son extrémité après leur remontée. Pour mettre au point cette approche bidimensionnelle, nous avons utilisé et détaillé dans le rapport d'avant projet établi par GIC TUNISIE (2009), les équations développées par Stokes, Bonnefille, Mullenhoff et la théorie d'Airy relative à la trajectoire des particules et avons établi un modèle 2D que nous avons appelé MEDUSE pour définir les composantes de l'émissaire : longueur, diffuseur, efforts, etc.

\subsection{Oscillations de la houle}

Il est supposé que l'on dispose des données relatives aux paramètres $\mathrm{C}_{0}, \mathrm{~L}_{0}, \mathrm{~T}_{0}$ et $\mathrm{H}_{0}$, la célérité, la longueur d'onde, la période et la hauteur des vagues au large. Ensuite, en fonction du rapport «profondeur d'eau/longueur d'onde » (d/L), on peut déterminer les rapports $\mathrm{H} / \mathrm{H}_{0}$ et $\mathrm{L} / \mathrm{L}_{0}$ par les équations de BONNEFILLE (1976), et accéder aux valeurs de $\mathrm{H}$ et $\mathrm{L}$ puis à la profondeur $\mathrm{d}$ de l'eau. Ainsi pour le golfe de Tunis et pour une période $\mathrm{T}_{0}$ de $10 \mathrm{~s}$ et une hauteur $\mathrm{H}_{0}$ de $2 \mathrm{~m}$, selon les sites retenus on trouve une longueur moyenne de 5 à $6 \mathrm{~km}$ dont le premier kilomètre est à protéger contre les effets du déferlement.

\subsection{Dilution de l'effluent en deux phases}

A sa sortie de l'émissaire et en considérant que l'eau a subi un traitement en accord avec les normes strictes tunisiennes, traitement qui en principe élimine toute forme de décantation à son extrémité sauf accident épuratoire, l'effluent subit une dilution en 2 phases selon 2 directions.

Phase de remontée verticale : elle provoque la création d'un «nuage» de matières en suspension d'une densité liée à la qualité de l'effluent de profondeur fonction du profil de densité du milieu marin; si les valeurs des densités s'égalisent, le nuage est bloqué au cours de sa remontée, mais peut progresser horizontalement entrainé par les courants. Phase de transport par les courants horizontale : la théorie de Stokes développée audelà du $1^{\text {er }}$ ordre met en évidence un transport de matière dans le sens de propagation des vagues ce qui accentue la dilution. Ces effluents mettent ainsi un certain temps avant d'atteindre le rivage. L'objectif du concepteur dans la définition de la longueur de l'émissaire est que la concentration finale des polluants au niveau du rivage soit plus faible que celles fixée par les normes environnementales de rejet des eaux épurées dans le milieu naturel. Dans le cas du golfe de Tunis les zones à protéger sont les plages de la cote Nord et la distance minimale de l'émissaire pour assurer une dilution acceptable est de $10 \mathrm{~km}$ environ, des tests de sensibilité ayant testé des distances de 5 à $25 \mathrm{~km}$. 


\section{Les modèles numériques}

Les logiciels qui permettent de modéliser les courants marins sont basés sur les équations de Navier-Stokes et requièrent des données concernant notamment la bathymétrie de la zone et sa géométrie. Ensuite, pour caler le modèle, ce sont les conditions aux limites qu'il s'agit de préciser aux frontières de la zone d'étude (les hauteurs d'eau et les vitesses). Ces conditions aux limites restent délicates à définir compte tenu du fait que les débits entrant et sortant du domaine d'étude dépendent de la météorologie, assez bien connue, mais aussi de la courantologie, dont les données sont généralement déficientes. Chercher à mettre au point un modèle numérique sophistiqué en l'absence de mesures de terrain fiables des paramètres hydrodynamiques, revient à mettre la charrue avant les bœufs.

\section{Conclusion}

Pourquoi engager du temps et de l'argent (car les modèles sont souvent très coûteux) vu qu'en l'absence de données efficientes, la modélisation est si peu fiable même si les mesures a posteriori peuvent être considérées comme des tests de sensibilité ; car il est clair que sans conditions aux limites fiables mesurées in situ, le calage du modèle reste aléatoire!

Vu qu'il n'est pas nécessaire d'obtenir des résultats avec une précision élevée lorsqu'on estime une profondeur ou une longueur d'émissaire (sur nos côtes on travaille avec une précision à $1 \mathrm{~km}$ près), et que les principales contraintes sont constructives et concernent la stabilité de l'ouvrage. Ainsi du point de vue de l'ingénieur conseil, ne serait-il pas préférable, au lieu de chercher à faire caler un modèle coûte que coûte sur la base de quelques mesures ponctuelles et occasionnelles, de commencer par créer un « observatoire des émissaires de la Méditerranée » pour collecter des informations sur le fonctionnement des émissaires réalisés dans notre fragile mare nostrum, donner des éléments sur les paramètres de diffusion et relatifs à l'impact réel sur la posidonie, évaluer les défaillances de ces ouvrages, centraliser l'information afin de créer une base de données de recommandations concrètes au concepteur pour les nombreux projets à venir, vu que la sauvegarde de l'environnement terrestre et marin est l'avenir de cette planète et de cette mer qui a vu naître la civilisation ? Idée émise à creuser .....

\section{Référence bibliographique}

BONNEFILLE R. (1976). Cours d'hydraulique maritime. Masson, Paris, 209 p.

GIC TUNISIE (2009). APS et APD de la réhabilitation du rejet en mer de Choutrana. 